\title{
Energy dissipation in a shear layer with suction*
}

\author{
Charles R. Doeringa) \\ Department of Mathematics, University of Michigan, Ann Arbor, Michigan 48109-1109
}

Edward A. Spiegel

Department of Astronomy, Columbia University, New York, New York 10027

Rodney A. Worthing

Department of Mathematics, University of Michigan, Ann Arbor, Michigan 48109-1109

(Received 13 September 1999; accepted 4 May 2000)

\begin{abstract}
The rate of viscous energy dissipation in a shear layer of incompressible Newtonian fluid with injection and suction is studied by means of exact solutions, nonlinear and linearized stability theory, and rigorous upper bounds. The injection and suction rates are maintained constant and equal and this leads to solutions with constant throughput. For strong enough suction, expressed in terms of the entry angle between the injection velocity and the boundaries, a steady laminar flow is nonlinearly stable for all Reynolds numbers. For a narrow range of small but nonzero angles, the laminar flow is linearly unstable at high Reynolds numbers. The upper bound on the energy dissipation rate-valid even for turbulent solutions of the Navier-Stokes equations-scales with viscosity in the same way as the laminar dissipation in the vanishing viscosity limit. For both the laminar and turbulent flows, the energy dissipation rate becomes independent of the viscosity for high Reynolds numbers. Hence the laminar energy dissipation rate and the largest possible turbulent energy dissipation rate for flows in this geometry differ by only a prefactor that depends only on the angle of entry. () 2000 American Institute of Physics. [S1070-6631(00)02108-5]
\end{abstract}

\section{INTRODUCTION}

Fluid problems involving sources and sinks appear in many disciplines and applications ranging from biological to physical. By way of illustration we mention two well-studied physical examples. Suction is used as a device to reduce drag on an air foil ${ }^{1}$ because it is believed that suction delays boundary layer separation and so reduces drag. The second example is accretion flow in astrophysics ${ }^{2}$ in which matter in disks is sucked into black holes or onto other condensed objects, including stars in formation. This flow becomes turbulent and the resulting dissipation is important in determining the luminosities of the disks. Though in this work we shall be studying an example that is far from being astrophysically realistic, there is an aspect of the astrophysical models that relates to an interesting theoretical problem of turbulence.

In Kolmogorov's theory of homogenous isotropic turbulence, it is assumed that as the viscosity tends to zero, the dissipation rate becomes independent of viscosity. In other words, when measured in units of the typical velocity and length scales in the systems, the dissipation (per unit mass) goes like $\mathrm{Re}^{0}$ where $\mathrm{Re}$ is a Reynolds number such as the one to be defined in the next section. In the same way, modeling of astrophysical disks leads to estimates on the dissipation rate that is likewise independent of molecular viscosity.

\footnotetext{
*This paper is dedicated to Robert Kraichnan on the occasion of his 72nd birthday.

a) Author to whom correspondence should be addressed; electronic mail: doering@math.lsa.umich.edu
}

Naturally, there has been a considerable experimental effort aimed at testing the validity of such general conclusions but, in such experiments, the influence of walls is sometimes pervasive.

For many cases of turbulent shear flows between very smooth walls, the empirical friction law ${ }^{3,4}$ (a.k.a. the law of the wall) implies that the turbulent dissipation at large Renyolds numbers is like $\mathrm{Re}^{0} /(\log \mathrm{Re})^{2}{ }^{5,6}$ On the other hand, when the walls are rough, the results are different as is shown, for example, by some recent experiments on Couette flow $^{7}$ with textured walls. In the latter experiments, at least, it appears that breaking up the boundary layer changes its role in determining the total dissipation and leads to a Kolmogorov type of scaling with finite, viscosity independent total dissipation.

While it is rather difficult to model the effects of roughness of the walls in a theoretical study, especially one that is analytic in large part, there is another way to modify the contribution of the boundary layer to the total dissipation. We may take a lesson from the drag reduction method and suck the boundary layer out to let the main body of the fluid assert itself more strongly. Accordingly, in this paper, we study the fluid dynamics of a simple flow with suction and compute its rate of dissipation. Indeed, we study this flow in various ways.

We use the energy stability method $^{8}$ which, as we shall explain, leads us into considerations of obtaining bounds on the dissipation rates in the flow considered. Thus, even though some of the quantities of interest in flows with suction cannot be computed in detail when the flows are turbulent, we may hope to obtain bounds on them by exact math- 
ematical analysis and these will be valid for turbulent flows as well as for any laminar ones, steady or unsteady.

The procedures for bounding such quantities as turbulent dissipation have developed considerably in recent years with much of the work in this subject proceeding along one of two similar looking approaches. Both of these start by splitting the flow fields into two components, a background field and a fluctuating field, with the main distinction being in the nature of the background fields and their roles in the extremizing procedures. One of the methods uses a mean field in the manner of the Reynolds decomposition ${ }^{4}$ as the background field. It was first formulated by Malkus, ${ }^{9}$ reformulated and carried out successfully by Howard, ${ }^{10,11}$ and applied widely and effectively by Busse. ${ }^{12}$ The other approach adopts a device of $\operatorname{Hopf}^{13}$ to let the background field play a more central role in the variational procedures. This method, developed by one of us with Constantin, ${ }^{14}$ has ties to the energy stability theory ${ }^{8}$ and it too has been applied to a number of fluid problems, in Refs. 15-20, for example. Kerswell $^{21}$ has elucidated a formal mathematical connection between these approaches.

Our interest here is in the specific fluid problem mentioned and we shall explain details of the procedures as needed in the course of their use. In Sec. II we formulate the problem to be studied, a plane Couette flow with suction on one wall and injection on the other. In Sec. III we apply various stability concepts, both linear and nonlinear, to the laminar solutions to determine when they are physically relevant and when turbulence is possible and/or likely. A rigorous implementation of those ideas via the (second) background field method is presented in Sec. IV where we derive explicit rigorous upper bounds on the energy dissipation rate as a function of the system parameters. In the concluding Sec. V we summarize and discuss these results in view of recent analysis and experiments for shear driven turbulence.

\section{FORMULATION OF THE PROBLEM}

\section{A. Boundary conditions and equations}

To investigate the influence of suction on shear flows, we consider a layer of an incompressible (unit density) Newtonian fluid with (constant) kinematic viscosity $\nu$ confined between parallel rigid planes separated by distance $h$. The bottom plate, at $y=0$, is stationary and the top one, at $y$ $=h$, moves with speed $U^{*}$ in the $x$ direction. In referring to top and bottom plates we establish the convention that the $y$ direction is vertical, though there is no gravity in the problem. The third direction has coordinate $z$ and the unit vectors are $\mathbf{i}, \mathbf{j}, \mathbf{k}$. The velocity field is designated as $\mathbf{u}(\mathbf{x})=\mathbf{i} u_{x}$ $+\mathbf{j} u_{y}+\mathbf{k} u_{z}$.

In addition to the shearing motion imposed by the boundaries, there is a uniform injection of fluid into the layer with speed (flux) $V^{*}$ on the top plane. We seek solutions with constant throughput by imposing the same exit speed as fluid is removed uniformly on the bottom plane. (More generally, it would be of interest to let the rate of outflow vary with $t, x$, and $z$.) The conditions at the rigid boundaries are thus

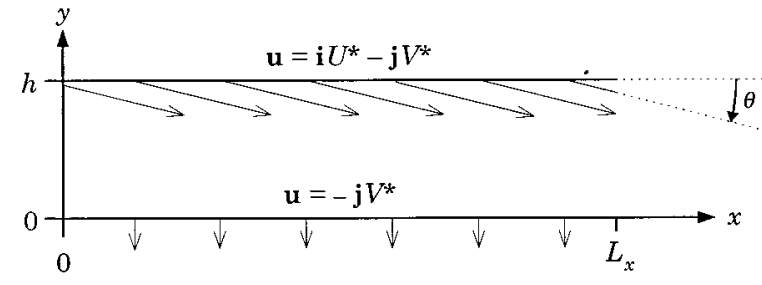

FIG. 1. Sketch of the boundaries and boundary conditions for the flows under consideration.

$$
\begin{aligned}
& \mathbf{u}=-\mathbf{j} V^{*} \text { at } y=0, \\
& \mathbf{u}=\mathbf{i} U^{*}-\mathbf{j} V^{*} \text { at } y=h,
\end{aligned}
$$

as illustrated in Fig. 1.

The velocity field and the pressure field $p(\mathbf{x}, t)$ are governed by the Navier-Stokes equations

$$
\begin{aligned}
& \frac{\partial \mathbf{u}}{\partial t}+\mathbf{u} \cdot \nabla \mathbf{u}+\nabla p=\nu \Delta \mathbf{u}, \\
& \nabla \cdot \mathbf{u}=0 .
\end{aligned}
$$

The incompressibility condition together with the boundary conditions leads to the supplementary boundary condition

$$
\frac{\partial u_{y}}{\partial y}=0 \text { at } y=0, h .
$$

In this work, we restrict our attention to periodic boundary conditions on all dependent variables in the horizontal directions with periods $L_{x}$ and $L_{z}$. The horizontal area of one cell in the layer is $A=L_{x} L_{z}$. In addition, we define the two control parameters of this problem, the Reynolds number

$$
\operatorname{Re}=\frac{h U^{*}}{\nu}
$$

and the entry angle, $\theta$, given by

$$
\tan \theta=\frac{V^{*}}{U^{*}} .
$$

We shall explore the stability characteristics and energy dissipation rates of various states in the $\operatorname{Re}-\theta$ plane.

\section{B. Energy dissipation}

Our focus throughout this paper is on the rate of energy dissipation per unit mass $\varepsilon$ as a function of $\nu, h, U^{*}$, and $V^{*}$. We shall derive an upper bound on $\varepsilon$ and compare it to the value associated with the laminar flow that we present in the next subsection. As we shall see, both the bound on $\varepsilon$ and its laminar value are nonvanishing and independent of kinematic viscosity $\nu$ as $\operatorname{Re} \rightarrow \infty$, and they are both finite and independent of viscosity in the limit of a semiinfinite fluid layer.

As the dissipation rate is a bulk property in the turbulent case, it is helpful in defining it to introduce the notation 


$$
\|f\|=\left(\int_{0}^{L_{x}} d x \int_{0}^{h} d y \int_{0}^{L_{z}} d z|f(x, y, z)|^{2}\right)^{1 / 2},
$$

which is the so-called $L_{2}$ norm, and

$$
\langle f\rangle=\limsup _{T \rightarrow \infty} \frac{1}{T} \int_{0}^{T} f(t) d t
$$

the largest possible value of the long time average of $f$.

The evolution equation for the kinetic energy in the fluid layer is

$$
\begin{aligned}
\frac{d}{d t} \frac{1}{2}\|\mathbf{u}\|^{2}= & \frac{1}{2} A V^{*} U^{* 2} \\
& +V^{*} \int_{0}^{L_{x}} d x \int_{0}^{L_{z}} d z\left[\left.p\right|_{y=h}-\left.p\right|_{y=0}\right] \\
& +\left.\nu U^{*} \int_{0}^{L_{x}} d x \int_{0}^{L_{z}} d z \frac{\partial u_{x}}{\partial y}\right|_{y=h}-\nu\|\nabla \mathbf{u}\|^{2},
\end{aligned}
$$

which follows from the Navier-Stokes equations with the help of the boundary conditions. The presence of a total derivative with respect to time instead of a partial derivative serves to emphasize that while the norm does depend on time it is a constant in space. The terms on the right hand side of this equation are, in order, the net flux of kinetic into the layer, the rate of work performed by the injection and suction processes, the power expended shearing the fluid layer, and finally, the power removed by viscous dissipation in the fluid. The instantaneous rate of viscous energy dissipation is thus identified as $\nu\|\nabla \mathbf{u}\|^{2}$.

We concentrate on the largest possible value of the long time average of this last term, the viscous energy dissipation rate per unit mass

$$
\varepsilon=\frac{\nu}{h A}\left\langle\|\nabla \mathbf{u}\|^{2}\right\rangle .
$$

For the semi-infinite layer $(h \rightarrow \infty)$, we will consider instead the energy dissipation rate per unit area

$$
\hat{\varepsilon}=\frac{\nu}{A}\left\langle\|\nabla \mathbf{u}\|^{2}\right\rangle .
$$

\section{Laminar solution}

A simple exact steady solution of the problem is the laminar flow $\mathbf{u}_{\ell}=U_{\ell}(y) \mathbf{i}-V^{*} \mathbf{j}$ with

$$
U_{\nearrow}(y)=U^{*} \frac{1-e^{-V^{*} y / \nu}}{1-e^{-\operatorname{Re} \tan \theta}} .
$$

For nonzero entry angles and values of Re not too small $(\operatorname{Re} \tan \theta \gg 1)$ the flow has a boundary layer at the suction plane, $y=0$. There the velocity deviates from a nearly constant bulk flow only in a layer of thickness

$$
\delta_{\ell}=\frac{\nu}{V^{*}}=\frac{h}{\operatorname{Re} \tan \theta} .
$$

Figure 2 shows streamlines for such laminar flows for sev-
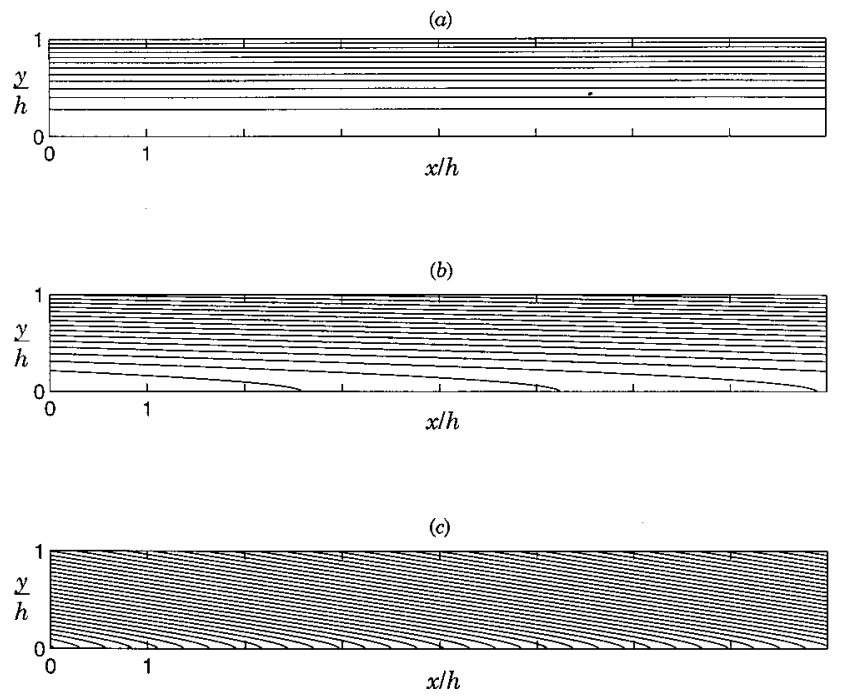

FIG. 2. Streamlines for the steady laminar flow at several parameter values. (a) Plane Couette flow, $\operatorname{Re}=100$ and $\theta=0$. (b) $\operatorname{Re}=99.99$ and $\theta=0.9^{\circ}$, with laminar boundary layer thickness $\delta / 0.64 h$. (c) $\operatorname{Re}=98.77$ and $\theta=9^{\circ}$, with laminar boundary layer thickness $\delta_{\ell} \approx 0.064 h$.

eral values of the injection angle, $\theta$.

When $\theta \rightarrow 0\left(V^{*} \rightarrow 0\right)$ the laminar solution reduces to plane Couette flow with $\mathbf{u}_{\ell}=\mathbf{i} U^{*} y / h$. This limit does not depend on the viscosity at all and retains its structure as $\nu$ $\rightarrow 0$. But for $\theta \neq 0$ and $y>0$, the vanishing viscosity limit is the constant flow field

$$
\lim _{\nu \rightarrow 0} \mathbf{u}_{\ell}=\mathbf{i} U^{*}-\mathbf{j} V^{*} \quad(\theta \neq 0) .
$$

This limiting velocity field has constant parallel flow in the bulk, is continuous at the injection boundary but is discontinuous at the suction boundary because $\mathbf{u} /(0)=0$ for all $\nu$ $>0$. The singularity is the result of $\delta, \rightarrow 0$ as $\operatorname{Re} \rightarrow \infty$ for fixed $\theta \neq 0$ and $h$; in general the limits $\theta \rightarrow 0$ and $\operatorname{Re} \rightarrow \infty$ do not commute as regards the velocity vector field.

The energy dissipation rate in the laminar solution is

$$
\begin{aligned}
\varepsilon_{\ell} & =\frac{\nu}{h} \int_{0}^{h}\left(\frac{\partial u_{x}}{\partial y}\right)^{2} d y \\
& =\frac{U^{* 2} V^{*}}{2 h} \frac{1}{\tanh ((1 / 2) \operatorname{Re} \tan \theta)} \\
& =\frac{U^{* 3}}{h} \frac{\tan \theta}{2 \tanh ((1 / 2) \operatorname{Re} \tan \theta)} .
\end{aligned}
$$

Not unexpectedly, this expression reduces to the energy dissipation rate in planar Couette flow as $\operatorname{Re} \rightarrow 0$ or $\theta \rightarrow 0$, namely

$$
\lim _{\operatorname{Re} \tan \theta \rightarrow 0} \varepsilon_{\ell}=\nu \frac{U^{* 2}}{h^{2}} .
$$

This dissipation rate vanishes as $\nu \rightarrow 0$, while for $\theta \neq 0$, the discontinuity in the flow at the suction boundary results in a residual dissipation in the limit of small viscosity 


$$
\lim _{\nu \rightarrow 0} \varepsilon_{\ell}=\frac{\tan \theta}{2} \frac{U^{* 3}}{h} \quad(\theta \neq 0) .
$$

Dimensional analysis implies that $\varepsilon$ is the cube of the velocity scale divided by a length scale in the system. This is the content of Eq. (2.18) with the prefactor depending on details of the geometry of the flow. We are familiar with scaling in which the energy dissipation remains finite and nonvanishing in the limit of high Reynolds number from Kolmogorov's theory of energy cascade in turbulence. This flow is an example of steady laminar flows in which the dissipation is finite in the zero viscosity limit because as the norm of the gradient goes up, the volume in which dissipation occurs goes down.

One of the main aims of this paper is to compare the high Re energy dissipation rate to an upper limit on the energy dissipation rate valid for any solutions of the NavierStokes equations, including turbulent solutions. One case where the Reynolds number becomes large is for fixed velocities and viscosity as the layer thickness increases. The semi-infinite layer is not a very interesting limit for $\theta=0$ at fixed $U^{*}$ because the rate of strain in Couette flow vanishes, whereas, for $\theta \neq 0$, the laminar solution for a deep layer with suction on the boundary has the structure

$$
\lim _{h \rightarrow \infty} \mathbf{u}_{\ell}=\mathbf{i} U^{*}\left(1-e^{-y / \delta}\right)-\mathbf{j} V^{*} \quad(\theta \neq 0) .
$$

The energy dissipation rate per unit horizontal area of the suction boundary is then

$$
\hat{\varepsilon}_{\ell}=\lim _{h \rightarrow \infty} \nu \int_{0}^{h}\left(\frac{\partial u_{x}}{\partial y}\right)^{2} d y=\frac{U^{* 2} V^{*}}{2}=\frac{\tan \theta}{2} U^{* 3},
$$

independent of viscosity.

\section{A useful decomposition}

A device that we shall use throughout this paper is to decompose the velocity field into a steady background flow and a time dependent field

$$
\mathbf{u}(\mathbf{x}, t)=\mathbf{i} U(y)-\mathbf{j} V^{*}+\mathbf{v}(\mathbf{x}, t),
$$

where the base horizontal flow profile $U(y)$ is as yet arbitrary except that it satisfies the boundary conditions on $u_{x}$. That is

$$
U(0)=0 \text { and } U(h)=U^{*} .
$$

The meaning of $\mathbf{v}$ will vary according to how we define $U$ but in all cases it satisfies the homogeneous boundary conditions

$$
\begin{aligned}
& \mathbf{v}=0 \text { at } y=0, \\
& \mathbf{v}=0 \text { at } y=h,
\end{aligned}
$$

together with periodicity in the horizontal.

Leaving aside the specification of $U$ for the present, we introduce the decomposition (2.21) into (3.1) and (3.2) to obtain

$$
\begin{aligned}
\mathbf{v}_{t}+\mathbf{v} & \cdot \nabla \mathbf{v}+\mathbf{i} v_{y} U^{\prime}(y)+\left[U(y) \partial_{x}-V^{*} \partial_{y}\right] \mathbf{v}-\mathbf{i} V^{*} U^{\prime}(y)+\nabla p \\
& =\nu \Delta \mathbf{v}+\mathbf{i} \nu U^{\prime \prime}(y),
\end{aligned}
$$

$\nabla \cdot \mathbf{v}=0$.

The evolution of the kinetic energy in the fluctuation field $\mathbf{v}$ is obtained by dotting $\mathbf{v}$ into Eq. (2.24) and integrating over the volume of a cell. Let

$$
\int_{\Omega} d \Omega=\int_{0}^{L_{x}} d x \int_{0}^{h} d y \int_{0}^{L_{z}} d z .
$$

Then on performing some integrations by parts and invoking the boundary conditions we obtain

$$
\begin{gathered}
\frac{d}{d t} \frac{1}{2}\|\mathbf{v}\|^{2}+\int_{\Omega} d \Omega U^{\prime}(y) v_{x} v_{y}-V^{*} \int_{\Omega} d \Omega U^{\prime}(y) v_{x} \\
\quad=-\nu\|\nabla \mathbf{v}\|^{2}-\nu \int_{\Omega} d \Omega U^{\prime}(y) \frac{\partial v_{x}}{\partial y}
\end{gathered}
$$

where $U^{\prime}$ is the derivative of $U$.

\section{STABILITY AND INSTABILITY}

The stability or instability of the steady laminar flow in (2.13) determines its realizability. Here we consider various aspects of the stability of our basic flow, both linear and nonlinear. From energy stability theory ${ }^{8}$ we shall see that the laminar flow is absolutely stable for sufficiently low Reynolds number or sufficiently large injection angles. On the other hand, we shall find that the laminar flow is unstable at high Reynolds numbers for sufficiently small (but nonzero) injection angles, thus merging into plane Couette flow, which is not linearly unstable at any Reynolds number, though it is nonlinearly unstable at large Reynolds number. ${ }^{22}$

\section{A. Energy stability}

For the general study of stability theory we let the background flow be the laminar solution, that is $U(y)=U /(y)$. Then the fluctuation equation (2.24) simplifies to

$\mathbf{v}_{t}+\mathbf{v} \cdot \nabla \mathbf{v}+\mathbf{i} v_{y} U^{\prime}(y)+\left[U_{\curlywedge}(y) \partial_{x}-V^{*} \partial_{y}\right] \mathbf{v}+\nabla p=\nu \Delta \mathbf{v}$.

The evolution equation of the kinetic energy in the fluctuation can now be written as

$$
\frac{d}{d t} \frac{1}{2}\|\mathbf{v}\|^{2}=-\mathcal{H}\{\mathbf{v}\}
$$

where the functional

$$
\mathcal{H}\{\mathbf{v}\} \equiv \int_{\Omega}\left[\nu|\nabla \mathbf{v}|^{2}+U_{\ell}^{\prime}(y) v_{x} v_{y}\right],
$$

is a homogeneous quadratic form in $\mathbf{v}$ that for any basic flow, $U_{\ell}$, associates a real number to each perturbation flow field. Equation (3.2), with (3.3), is known as the Reynolds-Orr energy equation. ${ }^{23}$

Energy stability theory rests on the observation that if the base flow is such that $\mathcal{H}$ is a positive quadratic form, then the kinetic energy in the fluctuation decays exponentially in time as $t \rightarrow \infty$, uniformly in the initial condition. Positivity of $\mathcal{H}$ means that there is a number $\mu>0$ such that

$$
\mathcal{H}\{\mathbf{v}\} \geqslant \mu\|\mathbf{v}\|^{2},
$$


for any divergence-free vector field $\mathbf{v}(\mathbf{x})$ satisfying the fluctuation's boundary conditions. When (3.4) is fulfilled, the kinetic energy satisfies the differential inequality

$$
\frac{d}{d t} \frac{1}{2}\|\mathbf{v}(\cdot, t)\|^{2} \leqslant-\mu\|\mathbf{v}\|^{2} .
$$

According to Gronwall's inequality (see Appendix A) this implies that the root-mean square fluctuation decays monotonically and exponentially:

$$
\|\mathbf{v}(\cdot, t)\| \leqslant\|\mathbf{v}(\cdot, 0)\| e^{-\mu t} .
$$

The domain of the $\theta-$ Re plane in which $\mathcal{H}$ is nonnegative is bounded by a curve of marginal energy stability. The location of this curve in the plane is determined by the level set $\mu(\theta, \operatorname{Re})=0$ where

$$
\mu(\theta, \operatorname{Re})=\inf \frac{\mathcal{H}\{\mathbf{v}\}}{\|\mathbf{v}\|^{2}},
$$

the infimum being taken over divergence-free vector fields satisfying the fluctuation's homogeneous boundary conditions.

\section{B. Absolute stability}

Without actually solving the variational problem for the curve of marginal energy stability, we may derive rigorous bounds on its location in several ways by analyzing the quadratic form directly. First we note that for any vector field that vanishes at $y=0$ and $y=h$, Poincare's inequality implies that $\|\nabla \mathbf{v}\|^{2} \geqslant\left(\pi^{2} / h^{2}\right)\|\mathbf{v}\|^{2}$. This is explained in Appendix $\mathrm{A}$, as is the fact that for divergence-free vector fields this estimate may be improved to

$$
\|\nabla \mathbf{v}\|^{2} \geqslant s \frac{\pi^{2}}{h^{2}}\|\mathbf{v}\|^{2},
$$

where the value $s=3.757 \cdots$ is obtained from the lowest eigenvalue of the Stokes operator in a slab. Bounds on the indefinite term in $\mathcal{H}$ may be found by using various of the inequalities given in Appendix A, and we find

$$
\left|\int_{\Omega} d \Omega U^{\prime}(y) v_{x} v_{y}\right| \leqslant \frac{1}{2} \frac{U^{*}}{h} \frac{\operatorname{Re} \tan \theta}{1-e^{-\operatorname{Re} \tan \theta}}\|\mathbf{v}\|^{2},
$$

where we used the fact that

$$
\sup _{y}\left|U^{\prime}(y)\right|=\frac{U^{*}}{h} \frac{\operatorname{Retan} \theta}{1-e^{-\operatorname{Re} \tan \theta}} .
$$

Combining these estimates we observe that

$$
\mathcal{H}\{\mathbf{v}\} \geqslant\left(\nu s \frac{\pi^{2}}{h^{2}}-\frac{1}{2} \frac{U^{*}}{h} \frac{\operatorname{Re} \tan \theta}{1-e^{-\operatorname{Re} \tan \theta}}\right)\|\mathbf{v}\|^{2},
$$

so that $\mathcal{H}$ is certainly positive if

$$
\frac{\operatorname{Re}^{2} \tan \theta}{1-e^{-\operatorname{Re} \tan \theta}}<2 s \pi^{2}=74.16 \cdots
$$

This condition corresponds to a curve $\operatorname{Re}_{1}(\theta)$ in the $\theta-\operatorname{Re}$ plane below which the steady laminar solution is absolutely

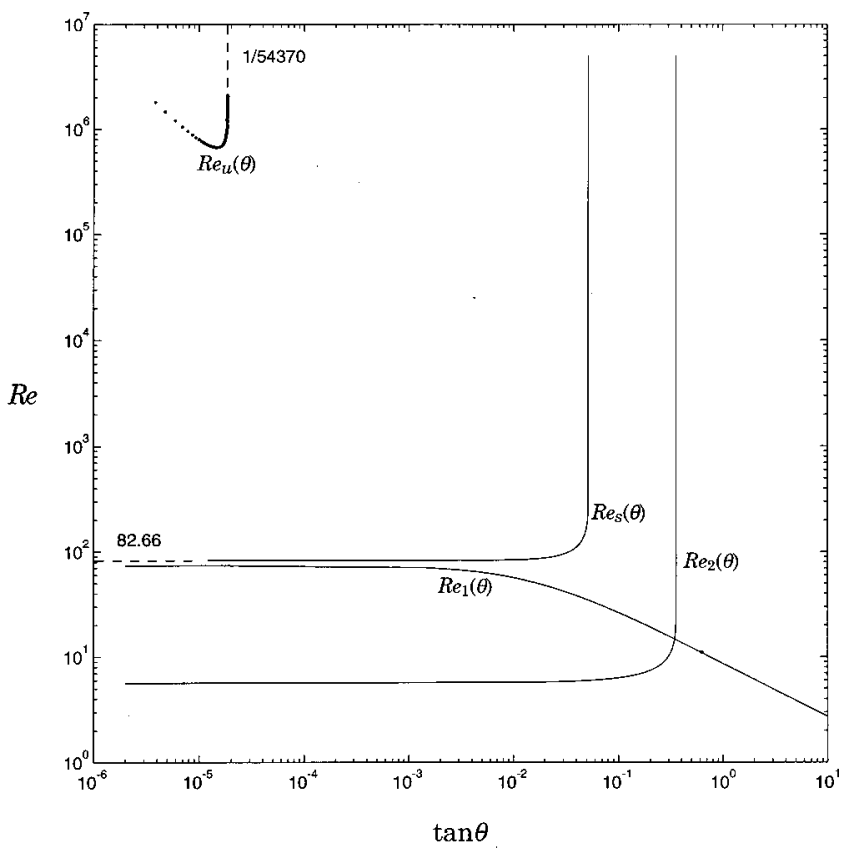

FIG. 3. Stability and instability boundaries in the $\theta-\operatorname{Re}$ plane. $\operatorname{Re}_{1}(\theta)$ and $\operatorname{Re}_{2}(\theta)$ are the rigorous energy stability boundaries derived in the text [the steady laminar flow is absolutely stable below $\operatorname{Re}_{1}(\theta)$ and to the right of $\left.\operatorname{Re}_{2}(\theta)\right] . \operatorname{Re}_{s}(\theta)$ is the numerically computed energy stability boundary; its horizontal asymptote is, as indicated, $2 \sqrt{1708} \approx 82.66$, the well known energy stability limit for planar Couette flow. Its vertical asymptote is at a critical injection angle of about $3^{\circ}$. The discrete points in the upper left hand corner lie on $\operatorname{Re}_{u}(\theta)$, the numerically computed curve of marginal linear stability. The steady laminar flow is unstable above $\operatorname{Re}_{u}(\theta)$ which has a vertical asymptote at the critical injection angle $\theta_{c} \approx \arctan \left(\frac{1}{54370}\right)$ $\approx 0.001^{\circ}$, the value for the semi-infinite layer computed by Hocking (Ref. 24).

stable. The curve from this estimate, shown as $\operatorname{Re}_{1}(\theta)$ in Fig. 3 , decreases from an absolute stability estimate for plane Couette flow, $\operatorname{Re}_{1}(0)=74.16 \cdots$. This proves that the laminar flow is absolutely stable if the Reynolds number is small enough.

A complementary bound on the location of the curve of marginal energy stability may be derived by focusing instead on the behavior of $\mathbf{v}$ near the suction boundary. Using standard analysis, we show, in Appendix B, that $\mathcal{H}$ is certainly positive if $\operatorname{Re}$ and $\theta$ satisfy

$$
1-\frac{\nu U^{*}}{2 \sqrt{2} \tan \theta}\left(1-\frac{\operatorname{Re} \tan \theta e^{-\operatorname{Re} \tan \theta}}{1-e^{-\operatorname{Re} \tan \theta}}\right)>0 .
$$

This condition corresponds to the line $\operatorname{Re}_{2}(\theta)$ in Fig. 3, below and to the right of the corresponding curve, the steady laminar solution is absolutely stable. The curve $\operatorname{Re}_{2}(\theta)$ has a vertical asymptote at $\tan \theta=\frac{1}{2} \sqrt{2}$. This proves that the laminar flow is absolutely stable if the injection angle $\theta$ $>\arctan \left(\frac{1}{2} \sqrt{2}\right) \approx 19.5^{\circ}$ no matter how high the Reynolds number.

The precise curve of marginal energy stability is determined by the solution of the variational problem in (3.7). We find it by deriving and solving the Euler-Lagrange equations of that problem. These are 


$$
\begin{aligned}
& \mu \mathbf{v}=-\nu \Delta \mathbf{v}+\nabla q+\frac{1}{2} U^{\prime}(y)\left(\mathbf{i} v_{y}+\mathbf{j} v_{x}\right), \\
& 0=\nabla \cdot \mathbf{v},
\end{aligned}
$$

where $q$ is the Lagrange multiplier from the incompressibility constraint and $\mathbf{v}$ satisfies the homogeneous boundary conditions. The critical Reynolds number of energy stability is determined, for a given injection angle $\theta$, by the smallest value of Re for which $\mu=0$.

These equations permit single Fourier modes in the $x$ and $z$ directions. From the variational statement, it can be shown that down-stream rolls $\left(\partial_{x}=0\right)$ are local extrema and numerical investigations confirm this. Such results may be obtained by using the numerical methods we shall describe in the following discussion of the linear problem. In that way, solutions were found for these equations. They determine the absolute stability boundary $\operatorname{Re}_{s}(\theta)$ in the $\theta-\operatorname{Re}$ plane shown in Fig. 3.

\section{Linear theory}

To address the issue of instability, we consider the linearized flow equations for $\mathbf{v}(\mathbf{x}, t)$

$$
\begin{aligned}
& \frac{\partial \mathbf{v}}{\partial t}+U_{\ell}(y) \frac{\partial \mathbf{v}}{\partial x}-V^{*} \frac{\partial \mathbf{v}}{\partial y}+\mathbf{i} U^{\prime}(y) v_{y}+\nabla p=\nu \Delta \mathbf{v}, \\
& \nabla \cdot \mathbf{v}=0 .
\end{aligned}
$$

For an $e^{-\lambda t} e^{i(\alpha x+\beta z)}$ dependence on $t, x$ and $z$ for all the variables, the problem reduces to the following ordinary differential equations for functions $v_{x}(y), v_{y}(y), v_{z}(y)$, and $p(z)$ :

$$
\begin{aligned}
& \lambda v_{x}-i \alpha U_{\ell}(y) v_{x}+V^{*} D v_{x}-U^{\prime}(y) v_{y}-i \alpha p \\
& \quad=\nu\left(-D^{2}+\alpha^{2}+\beta^{2}\right) v_{x}, \\
& \lambda v_{y}-i \alpha U_{\ell}(y) v_{y}+V^{*} D v_{y}-D p=\nu\left(-D^{2}+\alpha^{2}+\beta^{2}\right) v_{y},
\end{aligned}
$$

$\lambda v_{z}-i \alpha U_{\ell}(y) v_{z}+V^{*} D v_{z}-i \beta p=\nu\left(-D^{2}+\alpha^{2}+\beta^{2}\right) v_{z}$,

$i \alpha v_{x}+D v_{y}+i \beta v_{z}=0$,

where differentiation with respect to $y$ is denoted by $D$.

Linear instability occurs in the $\theta-\operatorname{Re}$ plane wherever the real part of an eigenvalue of $\lambda$ for some horizontal wave vector $\mathbf{i} \alpha+\mathbf{k} \beta$ is negative. The search for the boundary of this region, the condition of marginal stability, may be reduced to the equivalent two-dimensional stability problem with $v_{z} \equiv 0$ and $\beta \equiv 0$ by (a version of) Squire's transformation. $^{23}$ For any eigenvalue, $\lambda$, of the threedimensional problem at given values of $U^{*}, V^{*}, h, \nu$, and wave number $\mathbf{i} \alpha+\mathbf{k} \beta$, there is a lower (absolute) value of $U^{*}$ with the same eigenvalue $\lambda$ for the two-dimensional problem at another wave number $\mathbf{i} \tilde{\alpha}$. To see this, define

$$
\tilde{\alpha}=\sqrt{\alpha^{2}+\beta^{2}}
$$

and

$$
\tilde{\alpha} \tilde{v}_{x}=\alpha v_{x}+\beta v_{z} .
$$

Hence the three-dimensional incompressibility condition in Eq. (3.21) becomes a two-dimensional incompressibility condition

$$
i \tilde{\alpha} \tilde{v}_{x}+D v_{y}=0 .
$$

Then, adding together $\alpha \times(3.18)$ and $\beta \times(3.20)$ and dividing by $\tilde{\alpha}$ we observe that

$$
\begin{aligned}
& -\lambda \tilde{v}_{x}+i \tilde{\alpha}\left[\frac{\alpha}{\tilde{\alpha}} U_{\lambda}(y)\right] \tilde{v}_{x}-V^{*} D \tilde{v}_{x}+i\left[\frac{\alpha}{\tilde{\alpha}} U_{\ell}(y)\right] v_{y}+i \tilde{\alpha} p \\
& =\nu\left(D^{2}-\tilde{\alpha}^{2}\right) \tilde{v}_{x} .
\end{aligned}
$$

Recalling Eq. (2.13), we see that Eqs. (3.24) and (3.25) together with the transformed equation

$-\lambda v_{y}+i \tilde{\alpha}\left[\frac{\alpha}{\tilde{\alpha}} U_{\ell}(y)\right] v_{y}-V^{*} D v_{y}+D p=\nu\left(D^{2}-\tilde{\alpha}^{2}\right) v_{y}$,

are the two-dimensional equations with the substitution

$$
\left|U^{*}\right| \rightarrow\left|\frac{\alpha}{\tilde{\alpha}} U^{*}\right| \leqslant\left|U^{*}\right| .
$$

Thus, on taking $\beta=0=v_{z}$ in (3.18)-(3.21), we can eliminate $v_{x}$ and $p$ to produce a fourth order OrrSommerfeld-type equation for the normal velocity $v_{y}$

$$
\left\{\nu \mathcal{L}^{2}+i \alpha\left[U^{\prime \prime}(y)+U_{/}(y) \mathcal{L}\right]-V^{*} D \mathcal{L}\right\} v_{y}=\lambda \mathcal{L} v_{y},
$$

where

$$
\mathcal{L}=-D^{2}+\alpha^{2},
$$

and with boundary conditions $v_{y}=0=D v_{y}$ [because of (3.24)] at the walls.

A standard Chebyshev collocation technique in $y$ was employed to produce a matrix generalized eigenvalue problem for $\lambda$ which was then solved numerically. The numerically determined linear instability boundary $\operatorname{Re}_{u}(\theta)$ is also shown in Fig. 3. The laminar flow is unstable if the Reynolds number is high enough and the injection angle is small enough. The linearized instability boundary in the $\theta-\operatorname{Re}$ plane has a vertical asymptote that agrees with the analysis of Hocking ${ }^{24}$ for the semi-infinite layer. This correspondence serves as a check on the computation.

The laminar flow is linearly unstable at high Reynolds number for angles $0<\theta<\theta_{c}=\tan ^{-1}(1 / 54370) \approx 0.001^{\circ}$. The gaps in both angle and Reynolds number between the boundaries of absolute stability and linear instability, are consistent with Hocking's conclusion that the bifurcation in the semi-infinite layer at $\theta_{c}$ is subcritical.

\section{BOUNDS ON ENERGY DISSIPATION}

Let us first sketch an intuitive derivation of the kind of result to be anticipated. At high Reynolds numbers, we expect the dissipation to be dominated by the shear in a thin laminar sublayer. Let the Reynolds number of this boundary layer be $\operatorname{Re}_{\delta}=U^{*} \delta / \nu$ and suppose that it takes on a constant 
value, $r$, so that $\delta=r h /$ Re. If the dissipation takes place in the approximate volume $A \delta=(\delta / h)(A h)$, we can estimate that the mean dissipation rate is

$$
\begin{aligned}
\varepsilon & \approx \nu \frac{U^{* 2}+V^{* 2}}{\delta^{2}} \times \frac{\delta}{h} \\
& =\frac{\left(U^{* 2}+V^{* 2}\right) U^{*}}{r h}=\frac{1}{r \cos ^{2} \theta} \frac{U^{* 3}}{h} .
\end{aligned}
$$

Modulo the geometric prefactor, this is the same as the energy dissipation in the laminar solution. As an estimate for $r$ we may use the value of Re for marginal energy stability in the spirit of the proposal of Malkus ${ }^{25}$ to use the value for marginal linear stability.

\section{A. Upper bounds}

Now we turn to the derivation of upper bounds on the long-time averaged energy dissipation rate for any solution of the Navier-Stokes equations. We recall the decomposition of the velocity field described in Sec. II D but this time the background field is not specified in advance, as in stability theory, for instance. Instead, it is the method itself that leads to a determination of $U(y)$. In this, we are helped by the identity

$$
\begin{aligned}
\frac{\nu}{2}\|\nabla \mathbf{u}\|^{2}= & \frac{\nu}{2} A \int_{0}^{h} U^{\prime}(y)^{2} d y+\frac{\nu}{2}\|\nabla \mathbf{v}\|^{2} \\
& +\nu \int_{\Omega} d \Omega U^{\prime}(y) \frac{\partial v_{x}}{\partial y}
\end{aligned}
$$

which follows directly from the decomposition (2.21). As before, $\mathbf{v}$ satisfies the homogeneous conditions (2.23) and it varies according to (2.24) and (2.25). The evolution of the kinetic energy in the fluctuations is again (2.27).

We add Eqs. (2.27) and (4.2), multiply through by 2 and rearrange slightly, to obtain

$$
\begin{aligned}
\frac{d}{d t}\|\mathbf{v}\|^{2}+\nu\|\nabla \mathbf{u}\|^{2}= & \nu A \int_{0}^{h} U^{\prime}(y)^{2} d y-\nu\|\nabla \mathbf{v}\|^{2} \\
& -2 \int_{\Omega} d \Omega\left[U^{\prime}(y) v_{x} v_{y}-V^{*} U^{\prime}(y) v_{x}\right] .
\end{aligned}
$$

In this formula the last term is linear in $\mathbf{v}$, which is not desirable in the procedure. We may remove it by the transformation

$$
\mathbf{v}=\mathbf{w}-\mathbf{i} W(y),
$$

where $\mathbf{w}=\mathbf{i} w_{x}+\mathbf{j} w_{y}+\mathbf{k} w_{z}$ satisfies the fluctuation's homogenous boundary conditions. This substitution gives

$$
\|\nabla \mathbf{v}\|^{2}=\|\nabla \mathbf{w}\|^{2}-2 \int_{\Omega} d \Omega \quad\left(\partial_{y} w_{x}\right) W^{\prime}+\int_{\Omega} d \Omega \quad W^{\prime 2} .
$$

Then, on integration by parts of the middle term on the right, we see that it will cancel the unwanted last term in (4.3) if

$$
W^{\prime \prime}=-\frac{V^{*}}{\nu} U^{\prime}
$$

that is, if we choose

$$
W(y)=\frac{V^{*}}{\nu}\left[\frac{y}{h} \int_{0}^{h} U\left(y^{\prime}\right) d y^{\prime}-\int_{0}^{y} U\left(y^{\prime}\right) d y^{\prime}\right] .
$$

With the introduction of $\mathbf{w}$ into (4.3), we obtain

$$
\begin{aligned}
\frac{d}{d t} \frac{1}{A h} & \|\mathbf{v}\|^{2}+\frac{\nu}{A h}\|\nabla \mathbf{u}\|^{2} \\
= & \frac{\nu}{h} \int_{0}^{h} U^{\prime}(y)^{2} d y-\frac{2}{A h} \mathcal{I}\{\mathbf{w}\} \\
& +\frac{V^{* 2}}{\nu}\left[\frac{1}{h} \int_{0}^{h} U(y)^{2} d y-\left(\frac{1}{h} \int_{0}^{h} U(y) d y\right)^{2}\right],
\end{aligned}
$$

where

$$
\mathcal{I}\{\mathbf{w}\} \equiv \int_{\Omega} d \Omega\left\{\frac{\nu}{2}\|\nabla \mathbf{w}\|^{2}+U^{\prime}(y) w_{x} w_{y}\right\} .
$$

In effect, we have just completed the square in the integral in last term on the right hand side of Eq. (4.3). $\mathcal{I}$ is the same functional as $\mathcal{H}$ of stability theory, with $\nu$ replaced by $\nu / 2$ and $U^{\prime}$ instead of $U^{\prime}$.

Here is the essence of the bounding procedure: if the background profile may be chosen so that $\mathcal{I}$ is a non-negative quadratic form for divergence free vector fields satisfying the fluctuations' boundary conditions - as does the field $\mathbf{w}$ defined in Eqs. (4.4) and (4.7) — then the $\mathcal{I}$ term in Eq. (4.8) may be dropped at the expense of the equality sign. That is

$$
\begin{aligned}
\frac{d}{d t} \frac{1}{A h} & \|\mathbf{v}\|^{2}+\frac{\nu}{A h}\|\nabla \mathbf{u}\|^{2} \\
\leqslant & \frac{\nu}{h} \int_{0}^{h} U^{\prime}(y)^{2} d y \\
& +\frac{V^{* 2}}{\nu}\left[\frac{1}{h} \int_{0}^{h} U(y)^{2} d y-\left(\frac{1}{h} \int_{0}^{h} U(y) d y\right)^{2}\right] .
\end{aligned}
$$

The right-hand side of (4.10) does not depend on time and the time average of this equation is

$$
\begin{aligned}
\frac{1}{A h} \frac{\|\mathbf{v}\|^{2}}{T}+\frac{\nu}{A h} \frac{1}{T} \int_{0}^{T}\|\nabla \mathbf{u}\|^{2} d t \\
\leqslant \frac{\nu}{h} \int_{0}^{h} U^{\prime}(y)^{2} d y \\
+\frac{V^{* 2}}{\nu}\left[\frac{1}{h} \int_{0}^{h} U(y)^{2} d y-\left(\frac{1}{h} \int_{0}^{h} U(y) d y\right)^{2}\right] \\
+\left.\frac{1}{A h} \frac{\|\mathbf{v}\|^{2}}{T}\right|_{t=0} .
\end{aligned}
$$

The first term on the left-hand side is manifestly nonnegative and so may be dropped respecting the inequality, 


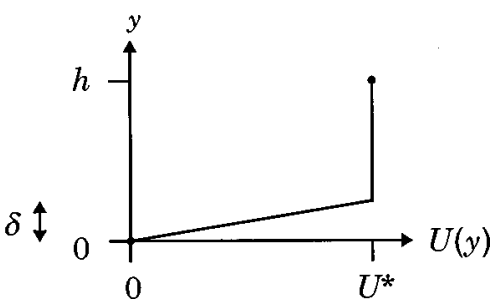

FIG. 4. Sketch of the background flow profile in Eq. (4.16).

and the last term on the right-hand side vanishes as $T \rightarrow \infty$ (since we assume finite initial kinetic energy). Hence, taking the long time limit, we deduce that

$$
\begin{aligned}
\varepsilon \leqslant & \frac{\nu}{h} \int_{0}^{h} U^{\prime}(y)^{2} d y \\
& +\frac{V^{* 2}}{\nu}\left[\frac{1}{h} \int_{0}^{h} U(y)^{2} d y-\left(\frac{1}{h} \int_{0}^{h} U(y) d y\right)^{2}\right] .
\end{aligned}
$$

This is an upper bound on $\varepsilon$ in terms of the background flow profile. In order to express $\varepsilon$ as an explicit function of the system parameters $U^{*}, V^{*}, \nu$ and $h$, we need to construct a background flow profile $U(y)$ satisfying the constraint that $\mathcal{I}$ is non-negative.

Since the quadratic form $\mathcal{I}$ is, apart from the change of $\nu$ into $\nu / 2$, the same as the quadratic form of energy stability theory in Eq. (3.3), the constraint that $\mathcal{I}$ be non-negative is equivalent to the condition that $U(y)$ be marginally energy stable at viscosity $\nu / 2$, as if it were a steady solution. Hence, if $U(y)$ were a steady, marginally energy stable profile for viscosity $\nu / 2$, it would lead to an upper bound on the largest possible energy dissipation rates according to the formula in Eq. (4.12), even for fully turbulent solutions of the NavierStokes equations.

On examining Eq. (4.11), we observe that $\mathcal{I}$ would be positive if $U^{\prime}(y)$ were to vanish, that is, if $U(y)$ was a constant across the layer. But that would be inconsistent with the requirement that the background profile satisfy the physical boundary conditions, $U(0)=0$ and $U(h)=U^{*}$. However, a choice where $U(y)$ is constant over most of the gap and varies only near the boundaries may work. This is because $w_{x}$ and $w_{y}$ must vanish at the boundaries so that the only way the product $U^{\prime}(y) w_{x} w_{y}$ can be large in magnitude is if $|\nabla \mathbf{w}|^{2}$ is also large. This leads to a successful strategy: Choose a background profile with a piecewise constant slope of the form

$$
U(y)=\left\{\begin{array}{c}
U^{*} \frac{y}{\delta} \text { for } 0 \leqslant y \leqslant \delta \\
U^{*} \text { for } \delta \leqslant y \leqslant h
\end{array},\right.
$$

with an adjustable boundary layer thickness $\delta$, as illustrated in Fig. 4.

To show that a small enough choice of $\delta$ ensures the non-negativity of $\mathcal{I}$ we may apply the analysis described in Appendix B to prove that

$$
\begin{aligned}
\left|\int_{\Omega} d \Omega U^{\prime}(y) w_{x} w_{y}\right| \leqslant & \frac{U^{*} \delta}{4 \sqrt{2}}\left[\left\|\frac{\partial w_{x}}{\partial y}\right\|^{2}+\left\|\frac{\partial w_{y}}{\partial y}\right\|^{2}+\left\|\frac{\partial w_{x}}{\partial x}\right\|^{2}\right. \\
& \left.+\left\|\frac{\partial w_{x}}{\partial z}\right\|^{2}+\left\|\frac{\partial w_{z}}{\partial x}\right\|^{2}+\left\|\frac{\partial w_{z}}{\partial z}\right\|^{2}\right] \\
\leqslant & \frac{U^{*} \delta}{4 \sqrt{2}}\|\nabla \mathbf{w}\|^{2}
\end{aligned}
$$

Now it is clear that the choice

$$
\delta=2 \sqrt{2} \frac{\nu}{U^{*}}
$$

ensures that $\mathcal{I}$ is non-negative. This choice makes sense for $\delta \leqslant h$, that is, for Reynolds numbers $\operatorname{Re} \geqslant 2 \sqrt{2}$. As we saw in the last section, the laminar flow is absolutely stable for values $\operatorname{Re}<2 \sqrt{2}$ where upper bounds are not of use. What remains now is to evaluate the upper bound in the parameter regime $\operatorname{Re} \geqslant 2 \sqrt{2}$.

Returning to Eq. (4.12) and adopting the background profile (4.13) with boundary layer thickness $\delta$ as in Eq. (4.15), we find

$$
\varepsilon \leqslant \varepsilon_{B} \equiv \frac{1}{2 \sqrt{2}} \frac{U^{* 3}}{h}+\frac{2 \sqrt{2}}{3} \frac{V^{* 2} U^{*}}{h}\left(1-\frac{3 \sqrt{2}}{2} \frac{\nu}{U^{*} h}\right) .
$$

This explicit upper bound on the energy dissipation rate is valid for any solution of the Navier-Stokes equations with these boundary conditions. In terms of the velocity scale $U^{*}$ and the injection angle $\theta$, the upper bound is

$$
\varepsilon_{B}=\frac{1}{2 \sqrt{2}}\left[1+\frac{8}{3} \tan ^{2} \theta\left(1-\frac{3 \sqrt{2}}{2} \frac{1}{\operatorname{Re}}\right)\right] \frac{U^{* 3}}{h},
$$

which, at high Reynolds numbers, becomes

$$
\varepsilon_{B} \sim \frac{1}{2 \sqrt{2}}\left(1+\frac{8}{3} \tan ^{2} \theta\right) \frac{U^{* 3}}{h} \quad \text { as } \operatorname{Re} \rightarrow \infty .
$$

Note also that the upper bound on the energy dissipation rate per unit horizontal area of the suction boundary, $\hat{\varepsilon}_{B}$ $\equiv \lim _{h \rightarrow \infty}\left(h \varepsilon_{B}\right)$ is finite in the limit of a semi-infinite fluid layer.

\section{B. Lower bounds}

We may also derive a lower limit to the energy dissipation as a function of the system parameters. However, the best rigorous estimate we can produce at this stage is well below the exact laminar dissipation rate, or indeed any estimates or bounds on the turbulent dissipation rate in the presence of suction at high Reynolds numbers. This issue raises questions with regard to lower bounds that center on the difficulty of imposing the Navier-Stokes equations as a constraint on the variational fields, as opposed to simpler functional constraints. For parameter values where the laminar solution is absolutely stable, it is clear that its dissipation rate represents the absolute minimum long time averaged dissipation rate among solutions of the Navier-Stokes equations. 
So the interesting parameter regime to study is that in which the exact laminar solution is not absolutely stable, that is, small $\theta$ and large Re.

Consider first the dissipation functional $\nu\|\nabla \tilde{\mathbf{u}}\|^{2}$ minimized over all divergence free vector fields-regardless of whether or not they are solutions of the Navier-Stokes equations-satisfying incompressibility and the boundary conditions. The Euler-Lagrange equations for this variational problem are just the steady Stokes equations with solution

$$
\mathbf{u}_{\text {Stokes }}=\mathbf{i} U^{*} \frac{y}{h}-\mathbf{j} V^{*} \text {. }
$$

Then it is easy to see that this solution realizes the global minimum of $\nu\|\nabla \tilde{\mathbf{u}}\|^{2}$. Indeed, let $\mathbf{v}$ be the difference between an arbitrary vector field $\mathbf{u}$ and the steady Stokes flow so that $\mathbf{u}(\mathbf{x})=\mathbf{i} U^{*}(y / h)-\mathbf{j} V^{*}+\mathbf{v}(\mathbf{x})$. Then use the homogeneous boundary conditions on $\mathbf{v}$ to find

$$
\nu\|\nabla \mathbf{u}\|^{2}=\nu \frac{U^{* 2}}{h^{2}} \times L_{x} h L_{z}+\nu\|\nabla \mathbf{v}\|^{2} .
$$

This expression shows explicitly that the dissipation rate in the Stokes flow is an absolute minimum to the dissipation rate for any flow. That is,

$$
\varepsilon \geqslant \varepsilon_{\text {Stokes }}=\nu \frac{U^{* 2}}{h^{2}}
$$

Note that this Stokes lower bound is independent of the injection angle (independent of $V^{*}$ ) and vanishes in the limit of zero viscosity. The exact laminar solution matches this dissipation only for $\theta=0$ or in the $\mathrm{Re} \rightarrow 0$ limit. The drawback of this lower bound is that Stokes flow is not a solution of the Navier-Stokes equation for nonvanishing $\theta$ and Re, so it is not realized by solutions, laminar or turbulent, of the Navier-Stokes equations.

We may investigate the minimization of $\nu\|\nabla \tilde{\mathbf{u}}\|^{2}$ over solutions of the Navier-Stokes equations by imposing those equations as constraints in a variational analysis. To illustrate the challenges that this endeavor presents, we consider just the stationary Navier-Stokes equations. Let us write the arbitrary solution of the steady Navier-Stokes equations as the steady laminar solution plus a variation

$$
\mathbf{u}=\mathbf{u}_{\ell}+\mathbf{v},
$$

where $\mathbf{v}$ is divergence free, satisfies homogeneous boundary conditions, and satisfies the transformed Navier-Stokes equations

$$
0=\left(U /(y) \partial_{x}-V^{*} \partial_{y}\right) \mathbf{v}+\mathbf{i} U_{\ell}^{\prime}(y) v_{y}+\mathbf{v} \cdot \nabla \mathbf{v}+\nabla p-\nu \Delta \mathbf{v} .
$$

We denote the Lagrange multiplier enforcing the divergence free constraint by $-2 q(\mathbf{x})$, and the Lagrange multiplier enforcing the Navier-Stokes equations by the divergence free field $2 \mathbf{w}(\mathbf{x})$ satisfying homogeneous boundary conditions. Then the functional to be minimized is the excess dissipation

$$
\begin{aligned}
\mathcal{F}\{\mathbf{v}\}= & \nu\|\nabla \mathbf{u}\|^{2}-\nu\|\nabla \mathbf{u} /\|^{2} \\
= & \int_{\Omega}\left\{\nu|\nabla \mathbf{v}|^{2}+2 \frac{\nu U^{*}}{\delta_{\ell}^{2}} \frac{e^{-y / \delta}}{1-e^{-h / \delta}} v_{x}-2 q \nabla \cdot \mathbf{v}\right. \\
& +2 \mathbf{w} \cdot\left[\left(U \varnothing(y) \partial_{x}-V^{*} \partial_{y}\right) \mathbf{v}+U^{\prime}(y) \mathbf{i j} \cdot \mathbf{v}\right. \\
& +\mathbf{v} \cdot \nabla \mathbf{v}-\nu \Delta \mathbf{v}]\} d \Omega .
\end{aligned}
$$

The Euler-Lagrange equations are the constraints 0 $=\delta \mathcal{F} / \delta q$ and $0=\delta \mathcal{F} / \delta \mathbf{w}$ together with

$$
\begin{aligned}
0=\frac{1}{2} \frac{\delta \mathcal{F}}{\delta \mathbf{v}}= & -\nu \Delta \mathbf{v}+\nabla q+\mathbf{i} \frac{\nu U^{*}}{\delta_{\ell}^{2}} \frac{e^{-y / \delta_{\ell}}}{1-e^{-h / \delta_{\ell}}} \\
& -\left(U_{\ell}(y) \partial_{x}-V^{*} \partial_{y}\right) \mathbf{w}+\mathbf{j} U^{\prime}(y) w_{x} \\
& -(\nabla \mathbf{w}) \cdot \mathbf{v}-\mathbf{v} \cdot \nabla \mathbf{w}-\nu \Delta \mathbf{w} .
\end{aligned}
$$

The steady laminar solution is an extremum of the dissipation functional if $\mathbf{v} \equiv 0$ solves these Euler-Lagrange equations. This does indeed solve the Euler-Lagrange equations when we choose the Lagrange multipliers according to $\mathbf{w}(\mathbf{x})=\mathbf{i} W(y)$ and $q(\mathbf{x})=q(y)$ with

$$
W^{\prime \prime}(y)-\frac{1}{\delta_{\ell}} W^{\prime}(y)=\frac{U^{*}}{\delta_{\ell}^{2}} \frac{e^{-y / \delta_{\ell}}}{1-e^{-h / \delta_{\ell}}}
$$

and

$$
q^{\prime}(y)=-U^{\prime}(y) W(y) .
$$

The solution for $W$ is

$$
W(y)=\frac{U^{*}}{2} \frac{\cosh \left[(y-h / 2) / \delta_{\ell}\right]-\cosh \left(h / 2 \delta_{\ell}\right)}{\sinh \left(h / 2 \delta_{\ell}\right)},
$$

and $q(y)$ follows by direct integration. Hence $\mathbf{u}_{\ell}$ is a critical point of the dissipation; it is a local minimum, a local maximum, or a saddle point.

With these choices for the Lagrange multipliers, the excess dissipation functional becomes

$$
\mathcal{F}\{\mathbf{v}\}=\int_{\Omega}\left[\nu|\nabla \mathbf{v}|^{2}-2 W^{\prime}(y) v_{x} v_{y}\right] d \Omega .
$$

This functional is similar in structure to the quadratic form of energy stability theory. In fact the coefficient of the indefinite term, proportional to $W^{\prime}(y)$, has a similar boundary layer structure for small $\delta_{\ell}$, now with boundary layers at both the injection and the suction boundaries. When the range of $\mathcal{F}$, defined on the restricted domain of $\mathbf{v}$ satisfying the transformed Navier-Stokes equations, is the set of nonnegative real numbers, then $\mathbf{u}_{\ell}$ is indeed the absolute minimum dissipation solution of the (steady) Navier-Stokes equations. But it is difficult to check the signature of $\mathcal{F}$ on that restricted functional domain. On the other hand it is straightforward to study $\mathcal{F}$ defined on the broader field of divergence-free $\mathbf{v}$ satisfying homogeneous boundary conditions, as in the context of energy stability theory. If $\mathcal{F}$ is a non-negative quadratic form on this extended domain then we may conclude that $\mathbf{u}_{\ell}$ is the absolute minimum dissipa- 
tion solution. However, an analysis like that for energy stability informs us only that $\mathcal{F}$ is non-negative for sufficiently small Reynolds number or sufficiently steep injection angles. That is, the analysis on the extended functional domain remains silent precisely in the dynamically interesting portion of the $\theta-$ Re plane where $\mathbf{u}_{\ell}$ is not absolutely stable.

This difficulty cannot be avoided by enlarging consideration to solutions of the time-dependent Navier-Stokes equations, $\mathbf{u}(\mathbf{x}, t)=\mathbf{u} \ell(\mathbf{x})+\mathbf{v}(\mathbf{x}, t)$. In that situation we introduce time-dependent Lagrange multipliers and an additional time integration. Presuming that we may neglect boundary terms from the time integrals, the variational problem is again solved by $\mathbf{v} \equiv 0$ and the same time independent Lagrange multiplier fields. The nature of the critical point still remains in question in the dynamically interesting region of the $\theta$ - Re plane.

It seems reasonable to expect that $\mathbf{u}_{\ell}$ is the minimum dissipation solution of the Navier-Stokes equations. We are unable to prove this conjecture with the mathematical technology at hand, but we may offer some more precise physical reasoning to that effect. Assuming that time averages $\langle\cdot\rangle$ of bulk quantities exist (the usual kind of assumption in statistical turbulence theory), we may deduce two identities from taking the space-time average of the streamwise and vertical components of the momentum equation

$$
\tau_{\text {bot }}=\tau_{\text {top }}+U^{*} V^{*}
$$

and

$$
\left\langle\int_{\mathrm{bot}} p d x d y\right\rangle=\left\langle\int_{\mathrm{top}} p d x d y\right\rangle,
$$

where bot and top refer to the planes at $z=0$ and $z=h$, and $\tau$ is the wall shear stress felt by the plates. Specifically, $\tau_{\text {top }}$ is the force per unit area in the $-\mathbf{i}$ exerted by the fluid on the top plate, and $\tau_{\text {bot }}$ is the force per unit area in the $+\mathbf{i}$ direction felt by the bottom plate. Combined with the time averaged energy balance from Eq. (2.10), we see that for any statistically stationary flow

$$
\varepsilon=\frac{1}{2} U^{* 2} \frac{V^{*}}{h}+\frac{U^{*}}{h} \tau_{\text {top }} .
$$

The first term on the right-hand side above is the net flux of kinetic energy into the system; the injection-suction boundary conditions input more kinetic energy than they extract. The second term on the right hand side is the power expended by the agent enforcing the slip boundary condition on the top; it is the work done by the top plate sliding on the fluid. Note that the first term from the kinetic energy flux is precisely the high Reynolds number limit of the dissipation in the exact steady laminar solution. Hence the only way that a turbulent energy dissipation rate could be less than the high Reynolds number limit of the laminar solution is if $\tau_{\text {top }}<0$. That would mean that, on average, the turbulent flow was conspiring to push the top plate along, rather than provide any resistance to the shearing motion. This observation provides some more motivation for the study of this lower bound problem. We shall not pursue the issue further here, leaving open the question of the minimum dissipation among

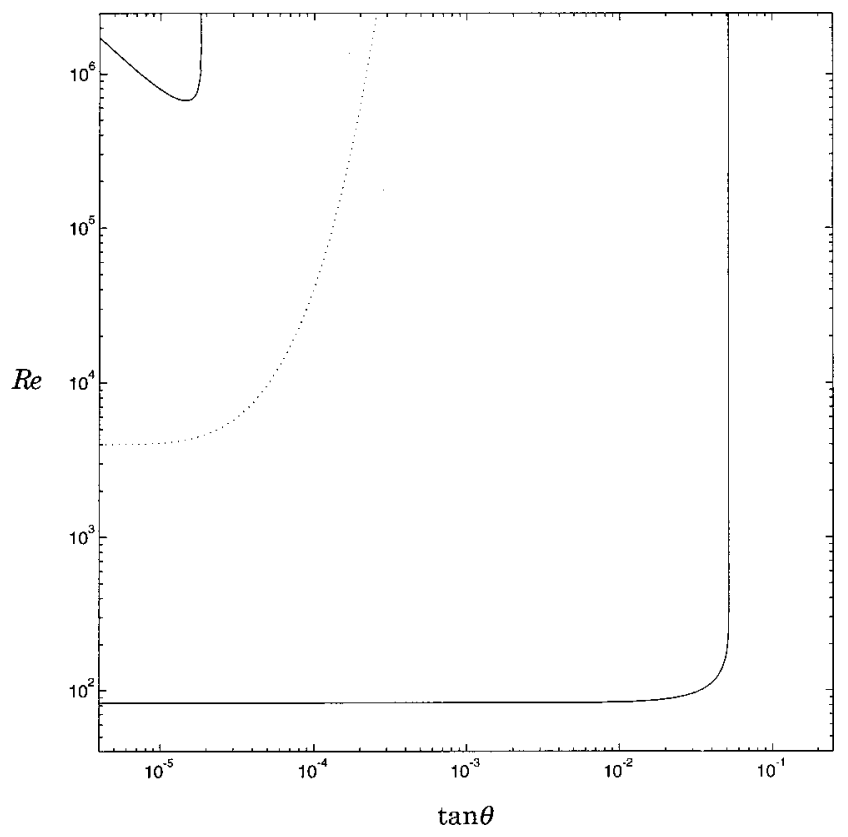

FIG. 5. Summary of the stability portrait in the $\operatorname{Re}-\theta$ plane. The steady laminar flow is absolutely stable according to the energy method for Re $<82$ or $\theta>3^{\circ}$ (with $\tan \theta \approx 0.05$ ). The steady laminar flow is linearly unstable in the indicated region in the upper left hand corner where $\theta$ $<0.001^{\circ}$ (with $\tan \theta \approx 0.0002$ ) and $\mathrm{Re} \geqslant 700000$. The dotted line is a sketch of the conjectured nonlinear stability boundary for the steady laminar flow.

solutions, steady, unsteady or turbulent, of the NavierStokes equations with shear, injection and suction boundary conditions.

\section{SUMMARY AND DISCUSSION}

Suction is generally regarded as a stabilizing influence on flows as measured by the standards of parallel flows with similar looking boundary layer profiles. ${ }^{23}$ But in the linear version of our problem we see a minor exception to this general rule. Plane Couette flow, which may be thought of as the limit of the suction flow for zero suction velocity, that is, $\theta \rightarrow 0$, is linearly stable. However, in the $\theta-\operatorname{Re}$ plane (reproduced in Fig. 5 for the purposes of this discussion), we found that the marginal curve for linear instability has a minimum at the large but finite value $\mathrm{Re} \approx 700000$. As the entry angle tends to zero, the curve of marginal stability turns upward and reaches $\operatorname{Re}=\infty$ at $\theta=0$ in agreement with standard results for plane Couette flow. The critical Reynolds number also grows large as $\theta$ increases from the minimum point, in keeping with earlier stability studies for the semi-infinite layer $^{23,24}$ for which the stability criterion is measured in terms of $\operatorname{Re}_{\delta} \equiv U^{*} \delta / \nu=\tan \theta$. We may regard this band of unstable input angles as a linear instability inflicted on Couette flow by suction.

As to the nonlinear theory, we found that the flow is absolutely stable for Re less than a value close to 82 and for $\theta$ greater than about $3^{\circ}$. This defines the narrow, semiinfinite strip in the $\theta-$ Re plane seen in Fig. 5. In the relatively small region of the strip above the parabolic-looking curve in the upper left hand corner of Fig. 5, we find linear instability. The question raised then is: in how much of the 


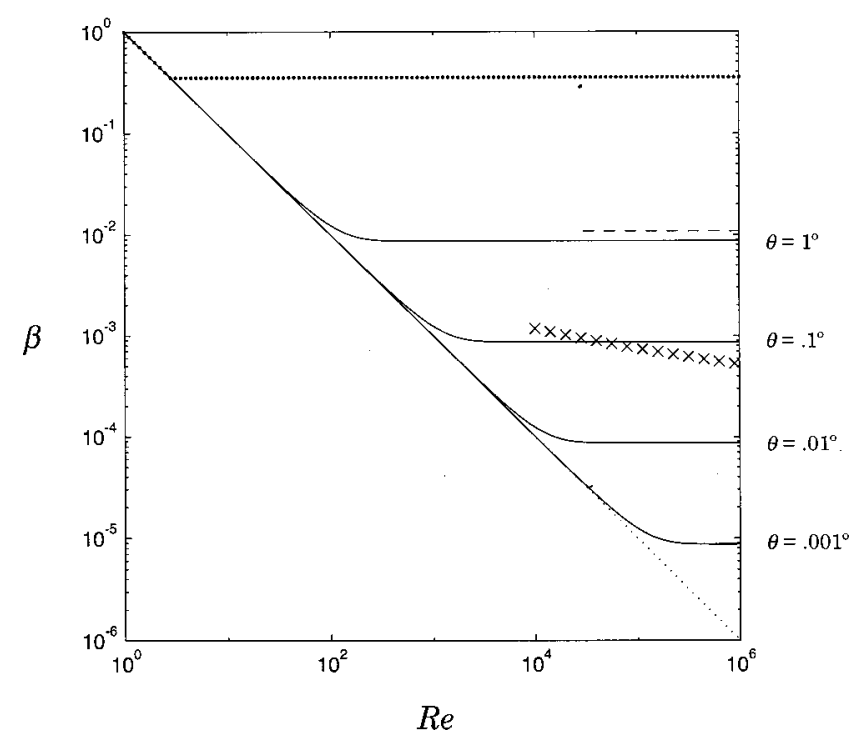

FIG. 6. The dissipation factor $\beta=\varepsilon \times h / U^{* 3}$ vs Reynolds number Re. The discrete data $(\cdots)$ at the top are the rigorous upper bound $\beta_{B}(\operatorname{Re}, \theta)$ in Eq. (5.2) for injection angles $\theta=1^{\circ}, 0.1^{\circ}, 0.01^{\circ}$, and $0.001^{\circ}$ (there is very little sensitivity of the bound to changes in $\theta$ at small angles). The dashed line segment (- - ) is the best known high Re bound for turbulent Couette flow, $\beta_{B}(\operatorname{Re}, 0) \approx 0.01087$ (from Ref. 26). The crosses $(\times)$ show the fit in Eq. (5.4) to experimental data. The solid lines (-) are, from top to bottom, the dissipation factor in Eq. (5.6) for injection angles $\theta=1^{\circ}, 0.1^{\circ}, 0.01^{\circ}$, and $0.001^{\circ}$. The lower envelope to the curves is the dissipation factor for plane Couette flow, the only rigorous lower bound available for the dissipation factor for arbitrary injection angles.

rest of this strip is the flow actually nonlinearly unstable? At $\theta=0$, nonlinear instability comes in at a Reynolds number (as measured here) of about $4000 .{ }^{22}$ We may expect that the onset of nonlinear instability occurs as one crosses upward through a curve in the $\theta$ - Re plane extending from the (nonlinear) marginal result for $\theta=0$. It is natural to imagine that this curve would go upward as $\theta$ is increased from zero in agreement with the usual notion that suction is stabilizing. Above this conjectured curve of nonlinear stability sketched as the discrete points in Fig. 5-the precise location of which remains to be computed-we expect to find nonlinear solutions that are the extension of the nonlinear instability of Couette flow.

The results on the bounds on dissipation also raise some intriguing issues. It is helpful to express the results in terms of the dimensionless dissipation factor

$$
\beta(\operatorname{Re}, \theta) \equiv \varepsilon \times \frac{h}{U^{* 3}} .
$$

The bound on $\varepsilon$ derived in Sec. IV corresponds to the rigorous upper limit

$$
\beta \leqslant \beta_{B}(\operatorname{Re}, \theta)=\frac{1}{2 \sqrt{2}}\left[1+\frac{8}{3} \tan ^{2} \theta\left(1-\frac{3 \sqrt{2}}{2} \frac{1}{\operatorname{Re}}\right)\right],
$$

effective in regions of the $\theta$-Re plane where the steady laminar solution is not absolutely stable. This bound allows for the residual dissipation in the vanishing viscosity limit in the sense that $\beta_{\ell} \rightarrow$ constant $>0$ as $\mathrm{Re} \rightarrow \infty$. In Fig. 6, the bound is plotted as a function of the Reynolds numbers for several small values of the injection angle. The dissipation factor bound in (5.2) is so insensitive to $\theta$ as $\theta \rightarrow 0$ that the points are not separated by more than the size of a dot.

In the $\theta \rightarrow 0$ limit of planar Couette flow boundary conditions, the dissipation factor bound in (5.2) is $\beta_{B}(\operatorname{Re}, 0)$ $\approx 0.354$, a much cruder bound than may be obtained by more delicate variational estimates. The best bound known to date for turbulent planar Couette flow is the result of an optimization procedure whose high Re asymptotic value is ${ }^{26}$

$$
\beta_{B}(\operatorname{Re}, 0) \approx 0.011,
$$

also indicated in Fig. 6. Without doubt there is room for quantitative improvement in the bound in (5.2), and it remains to be seen how an optimized bound will depend on $\theta$. It is not unlikely, however, that the $\theta=0$ bound in (5.3) is accurate for very small angles and high Re where the bounds are relevant.

As we mentioned at the outset, existing (smooth-wall) data for shear-driven turbulence in the absence of suction are reasonably well fit by Prandtl's logarithmic law of the wall for which ${ }^{6}$

$$
\beta_{\mathrm{PK}} \approx 0.04 \times \frac{1}{(\ln \operatorname{Re})^{2}} \quad \text { as } \mathrm{Re} \rightarrow \infty .
$$

This empirical relation is indicated in Fig. 6, too. In the absence of suction, the upper bound analysis apparently yields the correct leading order scaling $\left(\beta \sim \operatorname{Re}^{0}\right)$ while failing to produce the logarithmic corrections.

Planar Couette flow with dissipation factor

$$
\beta /(\operatorname{Re}, 0)=\operatorname{Re}^{-1},
$$

realizes the absolute lower bound for the energy dissipation in the absence of suction. This dissipation factor also minimizes the dissipation functional over all divergence free vector fields satisfying the physical boundary conditions, as we found in Sec. IV B. This rigorous lower bound is the lower envelope of the curves in Fig. 5, a full factor of Re below the upper bound at high Re.

The dissipation factor for the steady laminar solution of the Navier-Stokes equations is

$$
\beta_{\curlywedge}(\operatorname{Re}, \theta)=\frac{\tan \theta}{2 \tanh ((1 / 2) \operatorname{Re} \tan \theta)} .
$$

This is plotted for several injection angles in Fig. 6. It converges to the lower bound $\operatorname{Re}^{-1}$ as $\operatorname{Re} \rightarrow 0$ or $\theta \rightarrow 0$ and, in contrast to the behavior in the absence of suction, it has a residual dissipation in the limit $\operatorname{Re} \rightarrow \infty$ when $\theta \neq 0$

$$
\beta_{\ell}(\operatorname{Re}, \theta) \sim \frac{\tan \theta}{2} \gg \frac{1}{\operatorname{Re}} \quad \text { as } \quad \operatorname{Re} \rightarrow \infty .
$$

While the steady laminar flow remains an exact solution of the Navier-Stokes equations and hence provides a lower limit to any mathematically rigorous upper bound on the dissipation factor, we stress that it is not known to be an absolute lower bound on the dissipation among solutions of the Navier-Stokes equations for small injection angles and high Reynolds numbers. 
These results and observations may have interesting implications for the mathematical analysis of solutions of the Navier-Stokes equations. This system represents, as far as we can ascertain, a first example with flux across its boundaries in which a finite constant residual dissipation is evident in the upper bound analysis. Heretofore, the best estimate for the high Reynolds number friction factor in the presence of flux at the boundaries was Hopf's original (and very general) bound, $\beta<O\left(e^{\mathrm{Re}}\right)$, which is not of much use practically. The upper bounds derived in this paper establish that a finite asymptotic residual dissipation bound is possible in the presence of flux. However, because of the simplicity of the geometry in this case, it remains unclear whether more general finite residual dissipation bounds, like those Wang ${ }^{27}$ recently obtained for shearing boundaries in the high Reynolds number limit, can be derived when there is a normal component to the velocity at the boundaries.

More than this, for any angle $\theta \neq 0$, the dissipation factor for the steady laminar solution undercuts the high Re extrapolation of $\beta_{\mathrm{PK}}$ associated with the logarithmic friction law. We cannot generally rule out the possible existence of (turbulent) flows with lower dissipation factors than $\beta_{\ell}$, so we cannot rule out the possibility of corrections like those in $\beta_{\mathrm{PK}}$. We may, though, confidently exclude the possibility of rigorously proving the existence of such corrections in the asymptotic $\mathrm{Re} \rightarrow \infty$ limit because the result for the exact laminar flow is sufficient to establish the precise nature of the strict $\beta \sim \operatorname{Re}^{0}$ scaling in any upper bound. However, it seems unlikely that the energy dissipation rate in a high Reynolds number turbulent flow would be smaller than that in a steady laminar flow. This suggests that corrections to the high Reynolds number scaling such as the logarithmic friction law are in a very real sense delicate modifications of Kolmogorov type scaling with a nonvanishing but finite residual dissipation. An arbitrarily small perturbation of the boundary condition in the form of a small amount of suction appears sufficient to destroy the logarithmic corrections in the high Reynolds number limit.

This latter observation is not altogether surprising as it is known that turbulent shear flow over a rough boundary produces such simple scaling. ${ }^{5}$ In the case of pipe flow, the turbulent dissipation factor depends on the degree of boundary roughness much as the dissipation factor depends on the injection angle for the problem studied in this paper. The sensitivity of such (logarithmic) corrections to perturbations in the boundary conditions is also illustrated in the recent experiments of Cadot $e t$ al. $^{7}$ where an array of bumps on the walls in a turbulent Couette flow experiment resulted in a constant dissipation factor quite close to that in (5.3). There have been Taylor-Couette experiments with suction, ${ }^{28}$ but it remains to be seen whether high Reynolds number sheardriven turbulence experiments with suction can be performed in order to investigate these issues further. And there have been direct numerical simulations of channel flow with injection and suction at the walls, ${ }^{29}$ but the systematic computational study of turbulent energy dissipation a simple shear layer with injection and suction remains. These kinds of high Reynolds number problems are of interest for laminar flows as well. ${ }^{30}$
Finally, from the point of view of astrophysical applications, the results reported here seem intriguing. The striking effects that suction has on the stability of shear flows imply that there will be strong interactions between the inflows in accretion disks and the shear. These could cause intermittency in the turbulence whose dissipation is thought to produce the luminosities of the disks. There may be here a partial explanation of the marked time dependence of these luminosities. To investigate such possibilities, we need to allow more general exit conditions since there is the implication that the throughput will become variable. Though the present study is still far from including astrophysically realistic conditions, the results uncovered suggest interesting possibilities for such applications.

\section{ACKNOWLEDGMENTS}

This research was supported in part by awards from the U.S. National Science Foundation to the University of Michigan. This work was begun while the authors were resident at the 1998 Summer Program in Geophysical Fluid Dynamics at the Woods Hole Oceanographic Institution and we thank the director for that year, Neil Balmforth. We are happy to acknowledge Sandip Ghosal with whom we discussed a related (and as yet unresolved) problem, Joe Keller for an interesting discussion on the issue of lower bounds, and Tom Mullin for bringing Ref. 28 to our attention.

\section{APPENDIX A: USEFUL INEQUALITIES}

Here is a brief summary of the key inequalities used in the analysis:

Schwarz's inequality. For functions $f(\mathbf{x})$ and $g(\mathbf{x})$ which are square integrable on the domain $\Omega$

$$
\int_{\Omega} f g d \Omega \leqslant\|f\|\|g\| .
$$

Hölder's inequality. There are more general versions of Hölder's inequality, but in this paper we use only the fact that

$$
\int_{\Omega} f g d \Omega \leqslant \sup _{\mathbf{x} \in \Omega}|f(\mathbf{x})| \int_{\Omega}|g| d \Omega .
$$

Poincare's inequality. One version of Poincare's inequality states that for any square integrable function $f(\mathbf{x})$ satisfying Dirichlet boundary conditions on the domain $\Omega$

$$
\|f\|^{2} \leqslant \frac{1}{\lambda_{1}}\|\nabla f\|^{2}
$$

where $\lambda_{1}$ is the smallest eigenvalue of the negative Laplacian with Dirichlet boundary conditions on the domain. For a slab of thickness $h$, this lowest eigenvalue is at least as large as $\pi^{2} / h^{2}$ corresponding to a function with a $\sin (y / h)$ dependence on $y$ across the slab. A divergence free vector field $\mathbf{v}$ satisfying Dirichlet boundary conditions on the domain $\Omega$ obeys

$$
\|\mathbf{v}\|^{2} \leqslant \frac{1}{\sigma_{1}}\|\nabla \mathbf{v}\|^{2},
$$


where $\sigma_{1}$ is the smallest eigenvalue of the Stokes operator

$$
\sigma \mathbf{v}=-\Delta \mathbf{v}+\nabla p, \quad \nabla \cdot \mathbf{v}=0,
$$

with Dirichlet boundary conditions.

Gronwall's inequality. Consider a function $f(t)$ satisfying the differential inequality

$$
\frac{d f}{d t} \leqslant-\mu f .
$$

Multiply by the positive integrating factor $e^{\mu t}$ to see that

$$
0 \geqslant e^{\mu t} \frac{d f}{d t}+\mu e^{\mu t} f=\frac{d}{d t}\left(e^{\mu t} f\right) .
$$

Hence $e^{\mu t} f$ is a decreasing function of time, and for $t \geqslant 0$ it is bounded by its initial value $f(0)$. This means that

$$
f(t) \leqslant f(0) e^{-\mu t} .
$$

\section{APPENDIX B: PROOF OF (3.13)}

In this Appendix we sketch the derivation of some of the inequalities referred to in the text. We start with the fundamental theorem of calculus in the form

$$
v_{x}(x, y, z)=\int_{0}^{y} \frac{\partial v_{x}\left(x, y^{\prime}, z\right)}{\partial y^{\prime}} d y^{\prime},
$$

and use the Schwarz inequality to deduce

$$
\left|v_{x}(x, y, z)\right| \leqslant \sqrt{y}\left(\int_{0}^{h}\left|\frac{\partial v_{x}\left(x, y^{\prime}, z\right)}{\partial y^{\prime}}\right|^{2} d y^{\prime}\right)^{1 / 2} .
$$

Similarly,

$$
\left|v_{y}(x, y, z)\right| \leqslant \sqrt{y}\left(\int_{0}^{h}\left|\frac{\partial v_{y^{\prime}}\left(x, y^{\prime}, z\right)}{\partial y^{\prime}}\right|^{2} d y^{\prime}\right)^{1 / 2} .
$$

Again, the Schwarz inequality, along with the fact that $|a b|$ $\leqslant(1 / 2) c a^{2}+(1 / 2 c) b^{2}$ for any $c>0$, implies that the second term in the integrand in $\mathcal{H}\{\mathbf{v}\}$ in Eq. (3.8) is bounded according to

$$
\begin{aligned}
& \left|\int_{\Omega} d \Omega U^{\prime}(y) v_{x} v_{y}\right| \\
& \quad \leqslant \frac{1}{2} \int_{0}^{h} y\left|U_{C}^{\prime}(y)\right| d y\left\{c\left\|\frac{\partial v_{x}}{\partial y}\right\|^{2}+\frac{1}{c}\left\|\frac{\partial v_{y}}{\partial y}\right\|^{2}\right\} \\
& \quad=\frac{\nu U^{*}}{2 V^{*}}\left(1-\frac{\operatorname{Re} \tan \theta e^{-\operatorname{Re} \tan \theta}}{1-e^{-\operatorname{Re} \tan \theta}}\right)\left\{c\left\|\frac{\partial v_{x}}{\partial y}\right\|^{2}+\frac{1}{c}\left\|\frac{\partial v_{y}}{\partial y}\right\|^{2}\right\} .
\end{aligned}
$$

The incompressibility condition $\nabla \cdot \mathbf{v}=0$ and some integrations by parts utilizing the boundary conditions together with applications of the Schwarz inequality imply that

$$
\left\|\frac{\partial v_{y}}{\partial y}\right\|^{2} \leqslant\left\|\frac{\partial v_{x}}{\partial x}\right\|^{2}+\left\|\frac{\partial v_{x}}{\partial z}\right\|^{2}+\left\|\frac{\partial v_{z}}{\partial x}\right\|^{2}+\left\|\frac{\partial v_{z}}{\partial z}\right\|^{2} .
$$

Then, choosing $c=1 / \sqrt{2}$, we have

$$
\begin{aligned}
\left|\int_{\Omega} d \Omega U^{\prime}(y) v_{x} v_{y}\right| \\
\leqslant \frac{\nu U^{*}}{2 \sqrt{2} V^{*}}\left(1-\frac{\operatorname{Re} \tan \theta e^{-\operatorname{Re} \tan \theta}}{1-e^{-\operatorname{Re} \tan \theta}}\right) \\
\quad \times\left\{\left(\left\|\frac{\partial v_{x}}{\partial y}\right\|^{2}+\left\|\frac{\partial v_{y}}{\partial y}\right\|^{2}+\left\|\frac{\partial v_{x}}{\partial x}\right\|^{2}+\left\|\frac{\partial v_{x}}{\partial z}\right\|_{2}^{2}\right.\right. \\
\left.+\left\|\frac{\partial v_{z}}{\partial x}\right\|^{2}+\left\|\frac{\partial v_{z}}{\partial z}\right\|^{2}\right\} \\
\leqslant \frac{\nu U^{*}}{2 \sqrt{2} V^{*}}\left(1-\frac{\operatorname{Re} \tan \theta e^{-\operatorname{Re} \tan \theta}}{1-e^{-\operatorname{Re} \tan \theta}}\right)\|\nabla \mathbf{v}\|^{2} .
\end{aligned}
$$

Inserting this estimate into Eq. (3.8) we deduce

$\mathcal{H}\{\mathbf{v}\} \geqslant \nu\left[1-\frac{1}{2 \sqrt{2} \tan \theta}\left(1-\frac{\operatorname{Re} \tan \theta e^{-\operatorname{Re} \tan \theta}}{1-e^{-R \sin \theta}}\right)\right]\|\nabla \mathbf{v}\|^{2}$.

Hence $\mathcal{H}$ is certainly positive if $\operatorname{Re}$ and $\theta$ satisfy

$$
1-\frac{\nu U^{*}}{2 \sqrt{2} \tan \theta}\left(1-\frac{\operatorname{Re} \tan \theta e^{-\operatorname{Re} \tan \theta}}{1-e^{-\operatorname{Re} \tan \theta}}\right)>0 .
$$

In analyzing the functional $\mathcal{I}$ in Sec. IV A the procedure is the same with $U_{\ell}$ replaced by the profile $U(y)$ from Eq. (4.13).

${ }^{1}$ G. K. Batchelor, An Introduction to Fluid Dynamics (Cambridge University Press, Cambridge, 1969).

${ }^{2}$ J. Frank, A. King, and D. Raine, Accretion Power in Astrophysics, Cambridge Astrophysics Series, Vol. 21 (Cambridge University Press, Cambridge, 1992).

${ }^{3}$ L. Prandtl, "Ueber die ausgebildete Turbulenz," Proc. 2nd Intern, Cong. Appl. Mech. Zurich (1926), p. 62.

${ }^{4} \mathrm{H}$. Tenekes and J. Lumley, A First Course in Turbulence (MIT, Cambridge, MA, 1972).

${ }^{5}$ J. Nikuradse, "Strömungsgesetze in rauhen Rohren," VDIForschungsheft 361 (1933).

${ }^{6}$ D. Lathrop, J. Feinberg, and H. Swinney, “Transition to shear-driven turbulence in Couette-Taylor flow,' Phys. Rev. A 46, 6390 (1992).

${ }^{7}$ O. Cadot, Y. Couder, A. Daerr, S. Douady, and A. Tsinober, "Energy injection in closed turbulent flows: Stirring through boundary layers versus inertial stirring," Phys. Rev. E 56, 427 (1997).

${ }^{8}$ D. D. Joseph, "Stability of fluid motions," Springer Tracts in Natural Philosophy (Springer-Verlag, Berlin, 1976).

${ }^{9}$ W. V. R. Malkus, "Lectures on Turbulence,'” in Notes on the 1960 Summer Study Program in Geophysical Fluid Dynamics, edited by E. A. Spiegel (Woods Hole Oceanographic Inst., 1960), Vol. II, pp. 1-68.

${ }^{10}$ L. N. Howard, "Heat transport by turbulent convection," J. Fluid Mech. 17, 405 (1963).

${ }^{11}$ L. N. Howard, "Bounds on flow quantities," Annu. Rev. Fluid Mech. 4, 473 (1972).

${ }^{12}$ F. H. Busse, “The optimum theory of turbulence," Adv. Appl. Mech. 18, 77 (1978).

${ }^{13}$ E. Hopf, "Ein allgemeiner endlichkeitssatz der hydrodynamik," Math. Ann. 117, 764 (1941).

${ }^{14}$ C. R. Doering and P. Constantin, "Energy dissipation in shear driven turbulence,"' Phys. Rev. Lett. 69, 1648 (1992).

${ }^{15}$ C. R. Doering and P. Constantin, "Variational bounds on energy dissipation in incompressible flows: Shear flow," Phys. Rev. E 49, 4087 (1994).

${ }^{16} \mathrm{C}$. Marchioro, "Remark on the energy dissipation in shear driven turbulence," Physica D 74, 395 (1994).

${ }^{17}$ P. Constantin and C. Doering, "Variational bounds on energy dissipation in incompressible flows: II. Channel flow," Physica D 82, 221 (1995). 
${ }^{18}$ T. Gebhardt, S. Grossmann, M. Holthaus, and M. Löhden, "Rigorous bound on the plane-shear-flow dissipation rate,' Phys. Rev. E 51, 360 (1995).

${ }^{19}$ R. Nicodemus, S. Grossmann, and M. Holthaus, "Improved variational principle for bounds on energy dissipation in turbulent shear flow,', Physica D 101, 178 (1997).

${ }^{20} \mathrm{C}$. Doering and P. Constantin, "Variational bounds on energy dissipation in incompressible flows: III. Convection,'” Phys. Rev. E 53, 5957 (1996).

${ }^{21}$ R. R. Kerswell, "Unification of variational principles for turbulent shear flows: the background method of Doering-Constantin and the meanfluctuation method of Howard-Busse,'’ Physica D 121, 175 (1998).

${ }^{22}$ A. Cherhabili and U. Ehrenstein, "Finite-amplitude equilibrium states in plane Couette flow,'” J. Fluid Mech. 342, 159 (1997).

${ }^{23}$ P. Drazin and W. Reid, Hydrodynamic Stability (Cambridge University Press, Cambridge, 1981).

${ }^{24}$ L. M. Hocking, "Non-linear instability of the asymptotic suction velocity profile,', Q. J. Mech. Appl. Math. 28, 341 (1974).
${ }^{25}$ W. V. R. Malkus, "The heat transport and spectrum of thermal turbulence,' Proc. R. Soc. London, Ser. A 225, 196 (1954).

${ }^{26}$ R. Nicodemus, S. Grossmann, and M. Holthaus, "The background flow method. Part 1. Constructive approach to bounds on energy dissipation,', J. Fluid Mech. 363, 281 (1998).

${ }^{27} \mathrm{X}$. Wang, "Time averaged energy dissipation rate for shear driven flows,", Physica D 99, 555 (1997).

${ }^{28} \mathrm{~K}$. Min and R. Leptow, "Circular Couette flow with pressure-driven axial flow and a porous inner cylinder,' Exp. Fluids 17, 190 (1994).

${ }^{29}$ Y. Sumitani and N. Kasagi, "Direct numerical simulation of turbulent transport with uniform wall injection and suction,', AIAA J. 33, 1220 (1995).

${ }^{30} \mathrm{R}$. Temam and X. Wang, "Boundary layers associated with incompressible Navier-Stokes equations: The noncharacteristic boundary case" (in press). 